\title{
THE INFORMATIONAL ROLE OF DISTRIBUTION SYSTEMS IN THE MARKET ECONOMY*
}

\author{
Yasuhiro SAKAI \\ and \\ Keisuke SAKAKI \\ Institute of Social Sciences University of Tsukuba \\ Tsukuba Ibaraki 305 JAPAN
}

Key Words : Intermediary, Distribution Channels, Demand Uncertainty.

\begin{abstract}
This paper investigates whether and to what extent the presence of distributors affects the working and performance of the market economy. Working with a simple Stackelberg leader-follower model, we show that the entry of an informed distributor brings about not only extra distribution costs (the intermediation effect), but also acquisition of market information (the information effect). If the demand uncertainty is relatively large and hence the information effect overpowers the intermediation effect, then the market economy with distribution channels performs better than the distribution-less economy. Therfore, distributive intermediation should be a natural response to informational asymmetry.
\end{abstract}

\section{INTRODUCTION}

This paper is concerned with the informational role of distribution systems in the market economy. It seems that the present state of research on distribution systems is far from satisfactory. The problem of vital importance is why distrubutions such as wholesalers and retailers exist in the market economy. Such raison d'etre for distribution channels is our main concern in this paper.

In the traditional micro-economic analysis, producers and consumers are regarded as two

\footnotetext{
* We are indebted to Martin J. Beckmann, Hirotada Kohno, Takao Fukuchi, Yoshio Kimura, Akio Matsumoto, Hiroshi Ohta, and Masayoshi Maruyama for their helpful comments and discussions. Any shortcomings that may remain are of course ours. Partial financial help from the Ministry of Education, Grant-in-Aid C-03630001, is gratefully acknowledged.
} 
main actors, but distributors play a very minor part. Although there exist really a variety of distribution channels including wholesalers, retailers and many other forms of middlemen, there seems very little theory which explains why merchants must exist between manufacturers and customers. In our opinion, the market economy without distribution channels looks like human body without blood. The purpose of this paper is to fill in such a gap between theory and reality by exploring the role of distributors played in the market economy. ${ }^{1}$

Several functions are performed by distributors in moving products from makers to consumers. First, buying and selling activities by intermediaries such as wholesalers and retailers are necessary to eliminate the locational gap between buyers and sellers. This is what we may call the physical distribution function. Second, storage and inventory of goods by distributors minimize the time gap between manufactures and end users. Third, distribution channels are designed to collect market information through distributors. While these three functions of distributive intermediaries are equally important, this paper focuses on the third function, i. e., the informational role of distributors in the market economy. We believe that this is worthy of theoretical investigation because it has been an unduly neglected area. ${ }^{2}$

Our problem here is clearly related to the discussion by Greenhut and Ohta $(1976,1979)$ of vertical integration of successive monopolists or oligopolists. They show that merger between an input supplier and a final good producer brings about a lower price, a greater output and a welfare gain to the society. We may think of the presence of a distribution channel between the producer and the end user as a sort of vertical separation, a phenomenon opposite to vertical integration. It can be conjectured, therefore, that the entry of a distributor results in a higher price, a smaller output and a welfare loss to the society unless the distributor performs other compensating functions such as acquisition of market information and elimination of transaction costs. This paper intends to investigate those compensating functions from an informational point of view. ${ }^{3}$

While there is a gorwing number of papers dealing with the welfare implications of information acquisition and pooling, most of the existing literature has so far confined itself to the analysis of duopoly or oligopoly models without distributive intermediaries. Of particular interest are Basar and Ho(1974), Ponssard(1979), Vives(1984, 1987), Gal-Or(1985,

1. There are many markets that are characterized by informational differences between sellers and buyers. In the related area of financial intermediation such as stock exchanges, mutual funds and banks, a growing number of papers dealing with the rationale for the intermediary are now available. Among them are Leland and Dyle(1977) and Chanbell and Kracaw(1980). Compared with this, the analysis of distributive intermediation has been an underdeveloped area.

2. These three functions performed by market channels were comprehensively discussed by Cavens(1987).

3. For a more recent study in vertical integration, see Abiru(1988). 
1986), Sakai(1984, 1985, 1990, 1991), and Sakai and Yoshizumi(1991a, 1991b).

An application of stochastic oligopoly theory to distribution channels was attempted by Maruyama (1988) on the basis of an earlier work of Sakai (1984). This paper aims to go beyond those works by making comparison between the market economy with distribution channels and the one without, thus shedding a new light on the informational role of intermediation.

In the world of perfect competition and perfect information, there is no room to accommodate for distributors. Producers and consumers may meet each other face to face, causing no frictions throughtout trading. Presence of middlemen could represent a complication factor in the linkage between makers and consumers. Then the direct distribution system without intermediaries could be Pareto superior to any system with informed or ignorant middlemen : both producers and consumers could be better off in the absence of distributors.

When competition is not perfect and information is not available, the situation changes completely. There emerges a room for distributors to play an active part in the market economy. It is true that distribution margins imposed by distributors could still add extra costs in the distribution flow from manufacturers to end users (the intermediation effect). However, distributors may as well contribute to collecting market information, especially in the society where consumers' tastes are changeable (the information effect). Without help of distributors, manufacturers would have considerable difficulty to foresee consumers' demand. In order to evaluate the working and performance of distribution systems, therefore, it is necessary to take account of these two mutually opposing effects — the intermediation and information effects. In some situations, the intermediation effect may overwhelm the information effect, and hence distributive intermediaries may be regarded as redundant agencies in the flow of goods and services. In other situations, however, the reverse would be the case : the information effect would dominate the intermediation effect. Then distributors would play a critical role in enhancing efficiency and welfare in the society.

The theoretical framework we employ in this paper is a simple Stackelberg model in which the maker plays the role of a leader and the distributor acts as a follower. There is uncertainly on the demand side. The distributor aims to maximize the amount of distribution margins while the maker aims to maximize its profits taking account of the distributor's reactions. The resulting equilibrium is a Stackelberg equilibrium where every player, the marker or the distributor, has no incentive to deviate from the equilibrium once it is achieved.

It is natural to suppose that the distributor has a great advantage over the maker in acquiring demand information. To make our point quite sharp, let us suppose that the distributor is fully informed of demand information but the maker is an ignorant player. In such an extreme situation, both the intermediation and information effects aforementioned are very easily measured as will be seen later. We can show that the relative strength of the 
intermediation and information effects depends on the degree of demand uncertainty. In case the uncertainty is quite large, the information effect may overpower the intermediation effect, implying that distributive intermediation should develop as a natural response to informational asymmetry.

The remainder of this paper is organized as follows. In Section 2, we introduce a basic model of the producer and the consumer without intermediation, and compute equilibrium values in this system. Section 3 discusses how the introduction of the distributor into our model affects the efficiency and welfare of the economy. In Section 4, we show that the welfare implications of distributive intermediation depend on the relative strength of the intermediation and information effects. Concluding remarks are made in Section 5.

\section{A Simple Maker-Consumer Model under Demand Uncertainty}

The model we are going to analyze as a starting point is the following, simple monopoly model under demand uncertainty. On the production side, we have a monopolistic sector producing an output, $x$. On the consumption side, we have a continuum of consumers of the same type, which are represented by the inverse demand function

$$
p=H(x ; \alpha),
$$

where $p$ is the unit price of $x$, and $\alpha$ is a random variable indicating demand uncertainty. As usual, we assume that $H$ is a non-increasing function of $x$. We assume that there is no limit of capacity at the firm. Let $C(x)$ be the cost function of the maker. We suppose that $C(x)$ is non-decreasing and concave in $x$. Letting $\Pi$ denote the profit of the maker, we find

$$
\Pi(x ; \alpha)=H(x ; \alpha) x-C(x) .
$$

Note that although the maker knows the probability function of $\alpha$, it may or may not know a true value of $\alpha$ before making his output decision. In the world where consumers' tastes are constantly changing, the maker may have difficulty to acquire exact information of market demand. So it would be natural to consider and compare the following two regimes. The first regime we consider is the one in which the maker is ignorant of $\alpha$ (Regime 0 ). The second regime is the one in which the maker can know $\alpha$ (Regime $F$ ). These two regimes are depicted in Figure 1 where a black roof on any house indicates that the resident of the house obtains information.

What we want to show in this paper is that the analysis of informational differences between makers and distributors is central to the theory of distributive intermediation. In order to accomplish our mission, we find it useful to work with the special case in which the market demand is linear and the producer's cost is ignored. Specifically, let us assume that $H(x ; \alpha)=\alpha-x$ and $C(x)=0$. Then it would be a fairly easy job to calculate various equilibrium values in this maker-consumer model with demand uncertainty. ${ }^{4}$ 
THE INFORMATIONAL ROLE OF DISTRIBUTION SYSTEMS IN THE MARKET ECONOMY

REGIME O : THE MAKER IS IGNORANT.

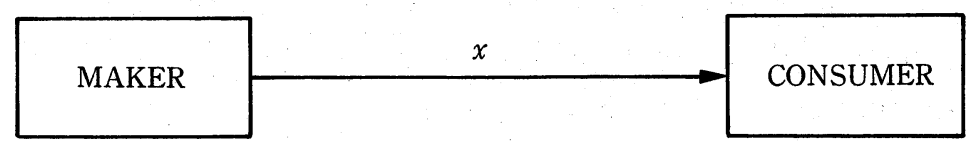

REGIME F : THE MAKER IS INFORMED.

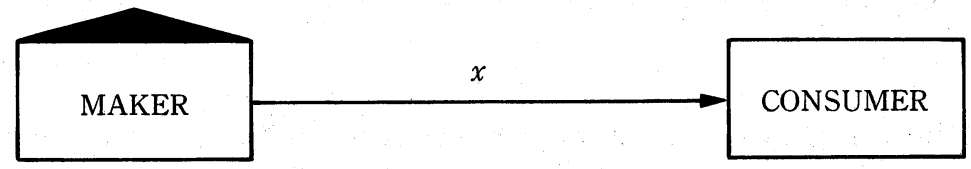

REGIME M : THE DISTRIBUTOR IS IGNORANT.

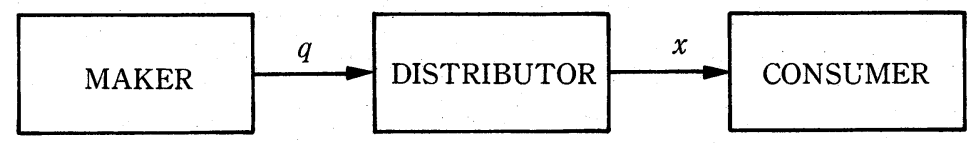

REGIME D : THE DISTRIBUTOR IS INFORMED.

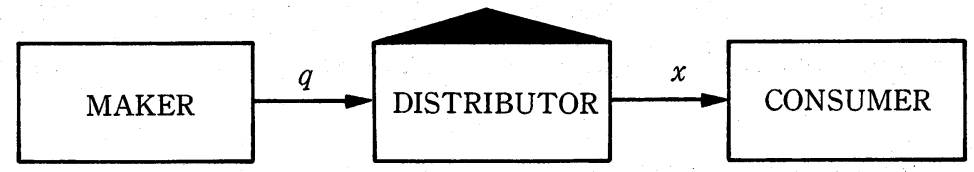

REGIME S : BOTH THE MAKER AND THE DISTRIBUTOR ARE INFORMED.

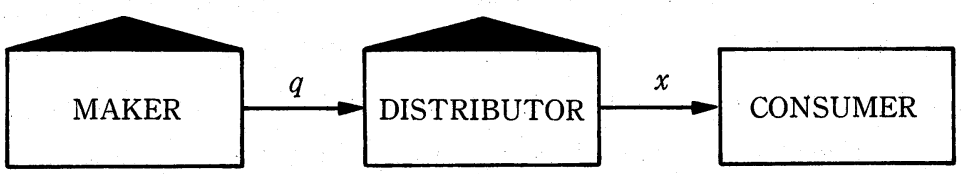

Figure 1. Distribution and Information: Alternative Regimes 
Under Regime 0 , the maker remains ignorant of $\alpha$ and is supposed to choose its output level to maximize its expected profit. Mathematically, we say that $x^{0}$ denotes an equilibrium output under Regime 0 if

$$
x^{0}=\arg \max _{x} \underset{\alpha}{E}[\Pi(x ; \alpha)] .
$$

Let $\mu$ stand for the mean of $\alpha$, i. e., $\mu=E \alpha$. Then since $\Pi(x ; \alpha)=x(\alpha-x)$ and $E \Pi(x$; $\alpha)=x(\mu-x)$ in the present regime, it follows that $x^{0}=\mu / 2, p^{0}=\alpha-\mu / 2$, and $\Pi^{0}=p^{0} x^{0}=\mu(\alpha$ $-\mu) / 4$. Because distributors are absent, the expected producer surplus, $E P S^{0}$, is equal to $E \Pi^{0}=\mu^{2} / 4$.

Consumer surplus under Regime 0 is measured by $C S^{0}=x^{0}\left(\alpha-p^{0}\right) / 2=\left(x^{0}\right)^{2} / 2$. Therefore, the corresponding expected consumer surplus and expected total surplus are respectively given by $E C S^{0}=\mu^{2} / 8$ and $E T S^{0}=E P S^{0}+E C S^{0}=(3 / 8) \mu^{2}$.

If the maker is informed about $\alpha$ under Regime $F$, then the situation changes drastically. The maker's output decision is now a contingent action, depending on the values of $\alpha$. Finally, we say that $x^{F}(\alpha)$ is an equilibrium output under Regime $F$ if for any given $\alpha$,

$$
x^{F}(\alpha)=\arg \max _{x} \Pi(x ; \alpha) .
$$

Let $\sigma^{2}$ denote the variance of the demand parameter $\alpha$, namely, $\sigma^{2}=E(\alpha-\mu)^{2}$. Then it is not hard to compute various equilibrium values under Regime $F$. We summarize the final results for both Regimes 0 and $F$ in the second and third columns in Table 1.

\section{Entry of the Distributor}

We are now in a position to let a third party enter our theoretical framework. The focal point of investigation is whether intermediation may develop as a response to uneven distribution of demand information between makers and distributors.

We suppose that once the distributor enters as a middleman, the industry structure is described by a Stackelberg type leader-follower model. We especially assume that the maker plays the role of a leader and the distributor acts as a follower. ${ }^{5}$

As before, the maker remains to be ignorant of demand uncertainty unless the information is transmitted to it from a third party. The following three regimes of distributive intermediation will be considered here. First, we will inquire into the regime in which the

4. Even if we instead assume that $C(x)=c x+k$, where $c$ and $k$ are parameters, the following analysis will also be applicable. In such a case, we would only have to reinterpret $\alpha$ as the net demand intercept, i. e., the difference between the demand intercept and the constant marginal cost, $c$.

5. The welfare analysis of information acquisition and transmission in a Stackelberg market was conducted by Sakai(1984), Gal-Or(1987) and others. Maruyama(1988) applied Sakai's analysis to distribution channels. However, Maruyama failed to compare the market economy with distributors and the one without. 
THE INFORMATIONAL ROLE OF DISTRIBUTION SYSTEMS IN THE MARKET ECONOMY

Table 1. Equilibrium Values Under Alternative Regimes

\begin{tabular}{c|c|c|c|c|c}
\hline \multirow{2}{*}{$\begin{array}{c}\text { Equilibrium } \\
\text { Values }\end{array}$} & \multicolumn{2}{|c|}{$\begin{array}{c}\text { Absence of Distributive } \\
\text { Intermediaries }\end{array}$} & \multicolumn{3}{|c}{ Entry of the Distributor } \\
\cline { 2 - 6 } & Regime $O$ & Regime $F$ & Regime $M$ & Regime $D$ & Regime $S$ \\
\hline$E x$ & $\frac{1}{2} \mu$ & $\frac{1}{2} \mu$ & $\frac{1}{4} \mu$ & $\frac{1}{4} \mu$ & $\frac{1}{4} \mu$ \\
$E p$ & $\frac{1}{2} \mu$ & $\frac{1}{2} \mu$ & $\frac{3}{4} \mu$ & $\frac{3}{4} \mu$ & $\frac{3}{4} \mu$ \\
$E I$ & $\frac{1}{4} \mu^{2}$ & $\frac{1}{4} \mu^{2}+\frac{1}{4} \sigma^{2}$ & $\frac{1}{8} \mu^{2}$ & $\frac{1}{8} \mu^{2}$ & $\frac{1}{8} \mu^{2}+\frac{1}{8} \sigma^{2}$ \\
$E S$ & - & - & $\frac{1}{16} \mu^{2}$ & $\frac{1}{16} \mu^{2}+\frac{1}{4} \sigma^{2}$ & $\frac{1}{16} \mu^{2}+\frac{1}{16} \sigma^{2}$ \\
$E P S$ & $E P S^{o}\left(=\frac{1}{4} \mu^{2}\right)$ & $E P S^{o}+\frac{1}{4} \sigma^{2}$ & $E P S^{o}-\frac{1}{16} \mu^{2}$ & $E P S^{o}-\frac{1}{16} \mu^{2}$ & $E P S^{o}-\frac{1}{16} \mu^{2}$ \\
& & & & $+\frac{1}{4} \sigma^{2}$ & $+\frac{3}{16} \sigma^{2}$ \\
$E C S$ & $E C S^{o}\left(=\frac{1}{8} \mu^{2}\right)$ & $E C S^{o}+\frac{1}{8} \sigma^{2}$ & $E C S^{o}-\frac{3}{32} \mu^{2}$ & $E C S^{o}-\frac{3}{32} \mu^{2}$ & $E C S^{o}-\frac{3}{32} \mu^{2}$ \\
& & & & $+\frac{1}{8} \sigma^{2}$ & $+\frac{1}{32} \sigma^{2}$ \\
& & & & $E T S^{o}-\frac{5}{32} \mu^{2}$ & $E T S^{o}-\frac{5}{32} \mu^{2}$ \\
& & & & $+\frac{3}{8} \sigma^{2}$ & $+\frac{7}{32} \sigma^{2}$ \\
\hline
\end{tabular}

distributor who has entered as the link between the maker and the consumer has no information about demand (Regime $M$ ). Second, we will consider the regime where the distributor as an entrant is an informed player. Third, we will pick up the regime in which the informed distributor transmits the information to the maker so that both the maker and the distributor may become informed. These three regimes of distribution are depicted in Figure 1 above.

In the presence of the distributor the two prices of $x$ should be distinguished. They are : the producer or mill price, $q$, and the consumer price, $p$. The difference between $p$ and $q$ represents what we may call the distributive margin.

Let us begin our inquiry with Regime $M$. We model the Stackelberg duopoly game as follows. The maker as a leader chooses $q$ so as to maximize the expected profit, $E \Pi(q, x$, $\alpha)$. The profit of the distributor is provided by

$$
\Omega(x, q, \alpha)=(p-q) x=(\alpha-q-x) x .
$$

The distributor takes the producer's output strategy as given, and chooses $x$ so as to maximize the expected profit, $E \Omega$.

More formally, let the reaction function of the distributor for the maker's choice of $q$ be given by $R^{M}(q)=\arg \max _{q} E \Omega(x, q, \alpha)$. Then we say that the pair $\left(x^{M}, q^{M}\right)$ of output and producer price is an equilibrium pair under Regime $M$ if

$$
x^{M}=R^{M}\left(q^{M}\right)
$$


and

$$
q^{M}=\arg \max _{q} \underset{\alpha}{\operatorname{En}}\left(q, R^{M}(q), \alpha\right)
$$

In the present case, it is not hard to find that $R^{M}\left(q^{M}\right)=(\mu-q) / 2, q^{M}=\mu / 2$ and $x^{M}=\mu / 4$. Note that the expected producer surplus is now the sum of $E \Pi$ and $E \Omega$. The final results for various equilibrium values under Regime $M$ are summarized in the fourth column in Table 1 above.

Let us turn our attention to the more intriguing case that the distributor may obtain demand information. Then at equilibrium, the distributor's output decision, $q^{D}$, is no longer a routine action, but a contingent action depending on the true value of $\alpha$. So the distributor maximizes its profit, $\Omega(x, q, \alpha)$, conditional on $\alpha$, while like in Regime $M$, the producer takes the distributor's reaction function into account, and maximizes its expected profit, $E \Pi$.

More mathematically, let the reaction function of the informed distributor be $R^{D}(q, \alpha)=$ $\arg \max _{x} \Omega(x, q, \alpha)$. Then the pair $\left(x^{D}, q^{D}(\alpha)\right)$ is an equilibrium pair under Regime $D$ if

$$
x^{D}(\alpha)=R^{D}\left(q^{D}, \alpha\right) \text { for any } \alpha,
$$

and

$$
q^{D}=\arg \max _{q} \underset{\alpha}{E} \Pi\left(q, R^{D}(q, \alpha), \alpha\right) .
$$

Note that in the present case, we have $R^{D}(q, \alpha)=(\alpha-q) / 2, q^{D}=\mu / 2$ and $x^{D}(\alpha)=(2 \alpha-\mu) / 4$. The equilibrium values under Regime $D$ can be summarized in the fifth column of Table 1 .

Now suppose that the informed distributor transmits the information to the maker so that both players can know the true value of $\alpha$. Then both the distributor's output decision, $q^{s}$, and the maker's (producer) price decision, $x^{s}$, become contingent actions depending on $\alpha$. Then the reaction function of the informed distributor under Regime $S$ should be the same as the one under Regime $D$, whence $R^{s}(q, \alpha)=\arg \max _{x} \Omega(x, q, \alpha)$. The pair $\left(x^{s}(\alpha), q^{s}(\alpha)\right)$ is an equilibrium pair under Regime $S$ if for any $\alpha$,

$$
x^{s}(\alpha)=R^{s}\left(q^{D}, \alpha\right)
$$

and

$$
q^{s}(\alpha)=\arg \max _{q} \Pi\left(q, R^{s}\left(q^{D}, \alpha\right), \alpha\right)
$$

The derivations of equilibrium values for Regime $S$ are similar to those for the previous regimes. Since $R^{s}(q, \alpha)=(\alpha-q) / 2, q^{s}(\alpha)=\alpha / 2$ and $x^{s}(\alpha)=\alpha / 4$, we can summarize the final results in the last coulumn of Table 1 .

\section{Comparison of Alternative Regimes : Intermediation and Information effects}

In the above, we have investigated the working and performance of alternative regimes under demand uncertainty. The first two regimes, Regimes 0 and $F$, characterize direct 
distribution in the sense that makers directly distribute their products to consumers without help of intermediaries such as wholesalers and retailers. Equilibrium values under the remaining three regimes, Regimes $M, D, S$, indicate how and to what extent the entry of middlemen affects the welfare of the market economy.

What we want to do in this section is to make a sequence of comparisons of these five regimes, thus searching for the rationale for distributive intermediation in the market economy. Our hypothesis is that intermediaries may emerge as information-gathering agencies because they usually have a significant advantage over manufactures in collecting and processing the market information.

In the light of Table 1, we can immediately establish

Proposition 1 (Regime $F$ versus Regime 0)

(i) $E x^{F}=E x^{0}, E p^{F}=E p^{0}$.

(ii) $E \Pi^{F}>E \Pi^{0}, E C S^{F}>E C S^{0}, E T S^{F}>E T S^{0}$.

The messages of this proposition are quite clear. Suppose that the maker gathers information about $\alpha$. Then although both expected output and expected price remain unaffected, both the maker's expected profit and the expected consumer surplus must increase. This implies that Regime $F$ is Pareto-superior to Regime 0 . Note that $E \Pi^{s}-E \Pi^{0}$ $=\sigma^{2} / 4, E C S^{F}=\sigma^{2} / 8$ and $E T S^{S}-E T S^{0}=3 \sigma^{2} / 8$. Therefore, the greater demand uncertainty, the greater is the social welfare, which agrees with common sense.

If are compare the first column with each of the third, the fourth and the fifth column in Table 1, we may easily obtain

Proposition 2 (Regime $F$ versus Regimes $M, D$ and $S$ ) For any $j=M, D, S$, we have the following properties :

(i) $E x^{F}>E x^{j}, E p^{F}<E p^{j}$.

(ii) $E P S^{F}>E P S^{j}, E C S^{F}>E C S^{j}, E T S^{F}>E T S^{j}$.

Proposition 2 has some interesting interpretations concerning the effects of the entry of the distributor on the welfare of the society. According to Property (i), the presence of middlemen brings bout a decrease in expected output and an increase in expected consumer price. Therefore, as Property (ii) shows, it yields a welfare loss in terms of EPS, ECS and ETS.

Proposition 2 together with Proposition 1 tells us that if the maker is fully informed, there should be no room to accommodate for distributors. In the world of perfect information on the production side, makers and consumers may meet each other face to face, causing no 
friction throughout trading. The presence of middlemen would merely serve as a redundant agency. This suggests that informational differences between makers and distributors may be a prime reason that intermediation must develop.

Proposition 3 (Regime $M$ versus Regime 0)

(i) $E x^{M}<E x^{0}, E p^{M}>E p^{0}$.

(ii) $E P S^{M}<E P S^{0}, E C S^{M}<E C S^{0}, E T S^{M}<E T S^{0}$.

When the distributor enters as an ignorant middleman, it is seen in this proposition that expected output decreases and expected consumer price increases. This is due to the fact that distributive margins imposed by the distributor add unnecessary costs in the distribution flow of products from the manufacturer to the end user. Such an intermediation effect, which may be contrasted with the well-known case of vertical integration of firms, brings about a welfare loss for both the production and consumption sides. Therefore, insofar as the distributor does not contribute to information acquisition, it plays the role of an intruder and thus the market economy performs worse with intermediation.

Now suppose that the distributor has access to demand information. Then we can show that the presence of the intermediary enhances the welfare of the whole society.

Proposition 4 (Regime $D$ versus Regime $M$ )

(i) $E x^{D}=E x^{M}, E p^{D}=E p^{M}$.

(ii) $E P S^{D}>E P S^{M}, E C S^{D}>E C S^{M}, E T S^{D}>E T S^{M}$.

This proposition demonstrates the informational role played by the distributor as the link between the maker and the consumer. Suppose that the middleman can gather demand information through its market research activities. For simplicity, we assume that the search costs are negligible. Then although both expected output and expected consumer price remain unaffected, the distributor's expected profit increases (namely, $E \Omega^{D}$ is greater than $\left.E \Omega^{M}\right)$, and hence expected producer surplus increases. Moreover, since expected consumer surplus also goes up by the acquisition of demand information, it follows that Regime $D$ is Pareto superior to Regime $M$.

Comparison of Proposition 3 and 4 indicates that the entry of the distributor gives rise to two mutually opposing effects in the market economy. They are : the intermediation effect as a minus factor, and the intermediation effect as a plus factor. The question which would naturally arise at this point is in what circumstances one effect dominates the other. An answer to this question will be given by the following proposition. 
Proposition 5 (Regime $D$ versus Regime 0 )

(i) $E x^{D}<E x^{0}, E p^{D}>E p^{0}$.

(ii) $E P S^{D} \gtreqless E P S^{0}$ according as $\sigma^{2} / \mu^{2} \gtreqless 1 / 4$, $E C S^{D} \gtreqless E C S^{0}$ according as $\sigma^{2} / \mu^{2} \gtreqless 3 / 4$, $E T S^{D} \gtreqless E T S^{0}$ according as $\sigma^{2} / \mu^{2} \gtreqless 5 / 12$.

The proof of Proposition 5 is straightforward from Table 1, and may be omitted here. By taking a close look at this proposition, we are able to draw several welfare implications of the entry of distributive agencies who have an edge over manufactures in informationgathering activities. We first note that whether the distributor is informed or ignorant, its entry into the market structure leads to a fall in expected output, and to a rise in expected consumer price (see and compare Proposition 4 (i) and 5 (ii)). Evidently, these consequences are not helpful for the welfare of consumers unless something else is brought out by the entrant. We suggest that such a counterbalancing factor is explained by the informational role of the intermediary.

It is of utmost importance to see whether and to what extent the presence of informed intermediaries influences the welfare of all members of the society. As was conjectured above, the welfare results are dependent on the relative strength of the intermediation and information effects. In view of Table 1 above, we note that the following formulas hold :

$$
\begin{aligned}
& \triangle E P S \equiv E P S^{D}-E P S^{0}=-\mu^{2} / 16 \quad+\sigma^{2} / 4 \\
& \triangle E C S \equiv E C S^{D}-E C S^{0}=-3 \mu^{2} / 32 \quad+\sigma^{2} / 8 \\
& \triangle E T S \equiv E T S^{D}-E T S^{0}=\quad-5 \mu^{2} / 32 \quad+3 \sigma^{2} / 8 \\
& \text { intermediation information } \\
& \text { effects effects }
\end{aligned}
$$

In the above set of formulas, the first and second terms on the right-hand sides represent the intermediation and information effects, respectively. Since these two effects go in opposite directions, their relative strength determines the consequences. More specifically, everything depends on the degree of uncertainty that is measured by the ratio of $\sigma^{2}$ to $\mu^{2}$.

The welfare effects are summarized in Table 2. On the one hand, in case the demand uncertainty is large enough (i. e., the $\sigma^{2}-\mu^{2}$ ratio exceeds three fourth in our model), the entry of the informed distributor is beneficial to all members of the society because the positive information effect overwhelms the negative intermediation effect in terms of EPS, ECS and ETS. On the other hand, when the demand risk is small enough (namely, the $\sigma^{2}-\mu^{2}$ ratio is less than one fourth), the situation changes completely. Then the intermediation effect 
Table 2. The Welfare Effects of the Entry of the Inforetd Distributor : Regime $O$ versus Regime $D$

\begin{tabular}{cccc}
\hline $\begin{array}{c}\text { Degree of } \\
\text { Demand Uncertainty }\end{array}$ & $\triangle E P S$ & $\triangle E C S$ & $\Delta E T S$ \\
\hline$\frac{\sigma^{2}}{\mu^{2}}>\frac{3}{4}$ & + & + & + \\
$\frac{5}{12}<\frac{\sigma^{2}}{\mu^{2}}<\frac{3}{4}$ & + & - & + \\
$\frac{1}{4}<\frac{\sigma^{2}}{\mu^{2}}<\frac{5}{12}$ & + & - & - \\
$\frac{\sigma^{2}}{\mu^{2}}<\frac{1}{4}$ & - & - & - \\
\hline
\end{tabular}

Note. For any quantity $Z, \triangle E Z=E Z^{D}-E Z^{\circ}$.

dominates the information effect so much that the market economy with the informed middleman performs better than the distribution-less economy.

If the $\sigma^{2}-\mu^{2}$ ratio takes on an intermediate value between these two critical values (i. e., $\left.1 / 4<\sigma^{2} / \mu^{2}<3 / 4\right)$, then there emerges the third possibility that information acquisition by the middleman benefits the production-distribution side but it hurts the consumption side, thus showing the conflict of interest among the members of the society. Even in this intermediate case, if the risk is considerably large (viz., $5 / 12<\sigma^{2} / \mu^{2}<3 / 4$ ), then expected total welfare increases by the intermediation.

To sum up, where substantial information asymmetries exist between makers and distributors and where demand uncertainty is relatively large, the market economy may perform superbly with distributive intermediation. Therefore, demand risk and informational nonsymmetry may be two main reasons that intermediaries exist.

Finally, let us consider whether the informed distributor has an incentive to share the information with the maker. By comparing the fifth and sixth columns in Table 1, we are immediately led to establish.

Proposition 6 (Regime $S$ versus Regime $D$ )

(i) $E \Pi^{s}>E \Pi^{D}, E \Omega^{S}<E \Omega^{D}$.

(ii) $E P S^{S}<E P S^{D}, E C S^{S}<E C S^{D}, E T S^{S}<E T S^{D}$.

The distributor is initially assumed to have demand information. When the distributor conveys the information to the maker, it is seen in Property (i) that the information receiver benefits but the transmitter hurts. As a result, the distributor has no incentive whatever to share the information with the maker. ${ }^{6}$

6. This was first pointed out by Maruyama(1988). 
Property (ii) indicates that such an information sharing decreases EPS, ECS and ETS. So when the distributor has information, the market economy performs better if the makers continues to be ignorant: System $D$ works better than System $S$.

In the above, we have assumed that the distributor and the maker are both expected-profit maximizes and hence risk-neutral players. Such an assumption might not be so realistic, however. When the distributor displays stronger risk aversion than the maker, it might be the case that information sharing makes both the transmitter and the receiver of information better off. If such a risk spreading effect works effectiverly, we can conjecture that System $S$, would work better than System $D$. However, these problems of risk-aversion and risk spreading would not be pursued further in this paper. ${ }^{?}$

\section{Concluding Remarks}

In this paper we have been concerned with question of why distributors such as wholesalers, retailers and other agencies are indispensable players in the market game under demand uncertainty. It is our belief that the present state of research in the operation of intermediation is far from satisfactory. Transaction costs could partly explain intermediation, but their magnitude does not seem to be the sole cause. We suggest that informational differences between distributors and manufacturers may be a prime reason that intermediation develops.

We believe that in the Age of Uncertainty, understanding the informational role of distribution systems is important not only from theoretical perspective, but also from policy perspective. For one thing, the Japanese distribution system has been criticized domestically and internationally. From American point of view, a multi-layered distribution channel in the Japanese economy adds unnecessary costs in the pipe line from manufactures to end users. If, however, we consider the information effect along with the intermediation effect as we have discussed in this paper, we would have a more balanced view of how different the Japanese distribution system is from the U. S. counterpart. For another, in socialist countries like the former U. S. S. R. and Eastern European countries, central planning agencies failed to fully recognize the vital role of distributors as the link between producers and consumers. It would be a too naive view that a party boss or his close associates in Kremlin can know what an ordinary citizen in Siberia wants to buy.

It is worth mentioning that our welfare results have obtained in a specific Stackelberg leader-follower model with specific functional forms assumed in demand and cost functions.

7. The impact of risk aversion on information sharing has recently been analyzed by Sakai and Yoshizumi (1991a, 1991b). The application of their analysis to distribution systems will be an item to be discussed in a subsequent paper. 
Besides, the issues of information-gathering costs and risk aversion have been all but ignored. While some of our results may perhaps depend on these specific assumptions, we believe that most of them are fundamentally robust for a more general framework. In other words, although changing any of these specific assumptions would change the precise conditions under which the positive information effect aforementioned dominates the negative intermediation effect and the market economy is thus better served with the presence of distributors, the possibilities still remain.

There are other directions in which we can generalize our analysis on the role of distributive intermediation. First, we have regarded the maker as a leader and the distributor as a follower in our Stackelberg game. In reality, however, we sometimes observe that giant trade companies and large-scale supermarkets may assume control of small-scale manufactures. Second, although we have focused on the informational role of distributors in this paper, there exist other functions to be performed by them. While buying and selling activities by intermediaries eliminate the locational gap between sellers and buyers, storage and inventory of goods by distributors minimize the time gap between manu-factures and end users. In our opinion, these two functions are more or less interlocked with the informationgathering function we have intensively explored in this paper. Third, the number of firms in the industrial structure is limited to only two : one maker and one distributor. However important these problem might be, they will be left for further research.

\section{References}

Abiru, M., 1988, Vertical integration, variable proportions and successive oligopolies, Journal Industrial Economics, vol. 36, 315-325.

Basar, T., and Y. C. Ho., 1974, Informational properties of the Nash solutions of two stochastic nonzero-sum games, Journal of Economic Theory, vol. 7., 370-387.

Campbell, T. S., and W. A. Kracaw, 1980, Information production, market signalling, and the theory of financial intermediation, Journal of Finance, vol. 35, 863-882.

Cravence, D, W., 1987, Strategic Marketing, Homewood, Ill, Richard D. Irwin, Inc.

Gal-Or, E., 1985, Information sharing in oligopoly, Econometrica, vol. 53, 329-343.

Gal-Or, E., 1986, Information transmission-Courunot and Bertrand equilibria, Review of Economics Studies, vol. 53, 85-92.

Gal-Or, E., 1987, First mover disadvantages with private information, Review of Economics Studies, vol. 54, 279-292.

Greenhut, M. H., and H. Ohta, 1976, Related market conditions and interindustrial margers, American Economic Review, vol. 66, 267-277.

Greenhut, M. H., and H. Ohta, 1979, Vertical integration and successive oligopolies, American 
Economic Review, vol. 69, 137-141.

Leland, H., E., and D. H. Pyle, 1977, Informational asymmetries, financial structure, and financial intermediation, Journal of Finance, vol. 32, 371-387.

Maruyama, M., 1988, Economic Analysis of Distribution, Tokyo, sobunsha.

Ponssard, J. P., 1979, Strategic Role of information in demand function in an oligopolistic market, Management Science, vol. 25, 243-250.

Sakai, Y., 1984, The role of information in a Stackelberg-type duopolistic market : A linear case, mimeo., Institute of Social Sciences, University of Tsukuba.

Sakai, Y., 1985, The value of information in a simple duopoly model, Journal of Economics Theory, vol. 36, 36-54.

Sakai, Y., 1990, Information sharing in oligopoly : Overview and evaluation, Part I, Alternative models with a common risk, Keio Economic Studies, vol. 27, 19-41.

Sakai, Y., 1991, Information sharing in oligopoly : Overview and evaluation, Part II, Private risks and oligopoly models, Keio Economic Studies, vol. 28, 51-71.

Sakai, Y., and A. Yoshizumi, 1991a, The impact of risk aversion on information transmission between firms, Journal of Economics, vol. 53, 51-73.

Sakai, Y., and A. Yoshizumi, 1991b, Risk aversion and duopoly : Is information exchange always beneficial to firms ?, Pure Mathematics and Applications, vol. 2, 129-145.

Vives, X., 1984, Duopoly information equilibrium : Courunot and Bertrand, Journal of Economic Theory, vol. 34, 7-94.

Vives, X., 1987, Trade association, disclosure rules, Incentives to share information and welfare, mimeo., Department of Economics, University of Pennsylvania. 